\title{
Perceived treatment efficacy of the last experienced illness episode in a community sample in the Northern Province, South Africa
}

\author{
K. Peltzer \\ PhD \\ Department of Psychology \\ University of the North
}

\begin{abstract}
This study determined the perceived efficacy of various health care providers for the last illness episode patients had suffered. 104 African/Black participants (50 male and 54 female), chosen by quota sampling from the general public (urban population of Mankweng), Northern Province, were interviewed. The research design was a one-shot case study. Medical treatment $(68 \%)$ was the major choice of treatment for minor, major and chronic medical condition as well as psychological/mental disorders. The second most important treatment choice was the herbalist (19\%) for minor and chronic conditions as well as traditional illness, followed by the diviner $(9 \%)$ prophet or faith healer (4\%) and psychologist $(2 \%)$. Combined traditional or faith healing was utilized by $32 \%$ of the sample and combined western treatment by $68 \%$. Regarding the self-rated treatment efficacy for the last illness episode western treatment was generally perceived as more effective than traditional or faith healing. However, traditional or faith healing was considered almost as long lasting as western therapy.
\end{abstract}

health care in South Africa."

\section{Introduction}

Before the April 1994 elections the African National Congress (ANC) proposed the following in its National Health Plan (ANC 1994a: 15):

Traditional healing will become an integral and recognised part of health care in South Africa. Consumers will be allowed to choose whom to consult for their health care, and legislation will be changed to facilitate controlled use of traditional practitioners.

Soon after the elections, the new Government of National Unity formalised this policy in its Reconstruction and Development Plan (RDP) (ANC 1994b: 47f.): There are deep divisions, fueled by mutual suspicion and lack of communication, between traditional and other complementary healers and medical and social workers. This is not in the interest of people who use all types of healers.
The RDP must aim to improve communication, understanding and cooperation between different types of healers. Hopa, Simbaye and du Toit (1998: 8-14) have investigated the perceptions on integration of traditional and western healing in the new South Africa. Different stakeholders (psychiatrists, medical doctors, psychologists, traditional healers and consumers) favoured the formal cooperation option for the integration of the two health systems.

As noted in South Africa and shown in other African countries traditional and faith healers play an important role in health care (Peltzer 1998b: 49, Pretorius 1989: 101). This may be expressed by the fact that for many South Africans the first choice of treatment is the traditional healer (De Beer in Swartz 1986: 276f., Farrand 1984: 780, Louw \& Pretorius 1995: 41). Swartz (1996: 124) has indicated that there is a paucity of studies on the efficacy of traditional and faith healing. Besides that it will be important to identify how effective ordinary people perceive traditional or faith healing. Almost nothing is known about how effective people perceive traditional or faith healing to be, and yet it is probably an influential factor determining whether they will use or recommend such a therapy (Vincent \& Furnham 1994: 129). Freeman and Motsei (1992: 1183) stated that there are broadly three types of traditional healers available to South African consumers. First the traditional doctor or inyanga. This is generally a male who uses herbal and other medicinal preparations for treating disease. Second the dingaka (Sotho), usually a woman who operates within a traditional religious supernatural context and acts as a medium with the ancestral shades. Third the faith healer who integrates Christian ritual and traditional practices. In accordance with previous studies (Peltzer 1998a: 192, Peltzer in print, Vincent \& Furnham 1994: 129) it is predicted that western health care will be used for major, life threatening, and generative illnesses whilst traditional healing will be consulted for minor, psychological, 
chronic and "traditional" illnesses.

This study aims to determine the perceived efficacy of various health care providers for the last illness episode patients had suffered (Vincent \& Furnham 1994: 128). The specific objectives are: (1) to identify the type of last illness episode suffered, needing professional health care attention, (2) to find out about the type of therapy utililized for the last illness episode, and (3) to determine the perceived efficacy of the therapy in question for the last illness episode.

\section{Method}

\section{Sample}

There were 104 African/Black participants (50 male and 54 female) from the general public, the urban population of Mankweng is 11211 (South Africa Census 1996). It is called urban because of the presence of tertiary institutions. The mean age of the sample was 32.2 years $(S D=11.5)$, with a range from 18 to 65 years. Most were Northern Sotho (84\%), Tsonga (9\%), Tswana (2\%) and others $(5 \%)$. Most participants (48\%) had some secondary education: $24 \%$ completed matric, $14 \%$ had primary only, $11 \%$ some tertiary and $4 \%$ no education. Almost one-third $(19 \%)$ indicated that they were students, $27 \%$ were not working, $15 \%$ house wives, $19 \%$ semi-professionals, $13 \%$ professionals, and $7 \%$ unskilled labourers. Thirtyone were married, 63 single, 2 divorced and 6 widowed. Most socio-demographic data are representative of the population census in 1996 (Statistics South Africa 1998).

The majority ( $88 \%)$ had no current illness but almost half (49\%) admitted to having minor complaints and $5 \%$ believed they were seriously ill. However, 38 (37\%) had been seriously ill at some time in the past.

Sixty participants $(58 \%)$ had had some experience of one or more forms of nonwestern therapy: 49 (47\%) had visited a traditional healer, 53 (51\%) had consulted a faith healer.

\section{Questionnaire}

The questionnaire consisted of 3 sections: (1) questions about biographic data and about their experience of traditional or faith healing (12 items), (2) 2 items on the last illness episode requiring professional attention (name of illness and type of health care intervention), and (3) 7 items on the treatment efficacy for the particular iliness episode such as "How effective do you think the treatment was?" or "Do you think you will remain feeling better?" rated on a 5 point Likert scale from $1=$ Not very effective/ likely to $5=$ very effective/likely).

Short definitions of each of the five therapy forms were given in case some participants were not familiar with them.

\section{Procedure}

Face to face interviews using the above questionnaire was conducted by one male and one female research assistant at various social meeting points such as taxi rank, bus stop, supermarket, restaurants, hospital waiting rooms, and bank in Mankweng. Quota sampling was done using the population census of $55.3 \%$ women and the adult age categories; 21 30 years $33 \%, 31-40$ years $14 \%, 41-50$ years $10 \%$, and $51-60$ years $3 \%$ (below 21 years $40 \%$ ). Thus 29 men and 31 women were chosen in the age group 21-30 years, 11 men and 12 women in the age group $31-40$ years, 8 men and 9 women in the age group 41-50 years. and 2 men and 2 women in the age group 51-60 years. A one-shot case study research design was used to determine whether the last intervention in the form of professional health care had any effect upon the group of participants. Participants were assured that confidentiality would be maintained. Almost all $(94 \%)$ volunteered to be interviewed under informed consent. Although this was not a large sample there is no reason to think that is was unrepresentative.

\section{Results}

Table 1 indicates the health-seeking behaviour for the last illness episode requiring professional health care by treatment type.

\section{Table 1: Treatment by type in percent}

\begin{tabular}{|c|c|c|c|c|c|}
\hline Type of illness & 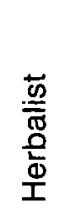 & 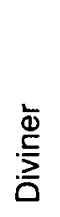 & $\begin{array}{l}\frac{\square}{\Phi} \\
\frac{\circ}{0} \\
\frac{0}{2}\end{array}$ & $\begin{array}{l}\overline{\mathscr{J}} \\
\bar{D} \\
\stackrel{\Phi}{\Sigma}\end{array}$ & 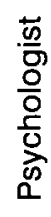 \\
\hline & \multicolumn{3}{|c|}{ Traditional } & \multicolumn{2}{|c|}{ Western } \\
\hline Major medical condition (cancer, major injury) & & & 2 & 3 & \\
\hline $\begin{array}{l}\text { Minor condition (gonorrhea, flue, headache, } \\
\text { stomach ache, knee pain, cystitis, stomach } \\
\text { ache, cold, eye problem, tonsillitis, dizziness, } \\
\text { skin problem, constipation, vomiting, body } \\
\text { pains, skin problems) }\end{array}$ & 10 & 4 & & 46 & \\
\hline $\begin{array}{l}\text { Chronic condition (allergies, ulcer, asthma, } \\
\text { epilepsy, back pain, hypertension) }\end{array}$ & 5 & 1 & 2 & 13 & \\
\hline Psychological (depression) & & 1 & & 4 & 2 \\
\hline Traditional (foot problem, spirit illness) & 5 & 4 & & 2 & \\
\hline All types & 20 & 10 & 4 & 68 & 2 \\
\hline
\end{tabular}

The major different conditions treated can be grouped into minor condition, chronic condition, traditional illnesses, psychological/mental disorder and major medical condition in that order of frequency. Medical treatment (68\%) was the major choice of treatment for minor, major and chronic medical condition as well as psychological/mental disorders. The second most important treatment choice was the herbalist $(19 \%)$ for minor and chronic conditions as well as traditional illness, followed by the diviner $(9 \%)$, prophet or faith healer $(4 \%)$ and psychologist (2\%). Combined traditional or faith healing was utilized by $32 \%$ of the sample and combined western treatment by $68 \%$.

The study partially confirmed the hypotheses that western health care will be used for major, life threatening, and generative illnesses whilst traditional healers were consulted for "traditional" illnesses and to a certain extent chronic illnesses. Contrary to the hypothesis, traditional and faith healing were not more used for minor condition and psychological problem.

Table 2 indicates the self-rated treatment efficacy by major type of therapy: traditional (herbalist, diviner and prophet) and western (medical personnel and psychologist)

Regarding the self-rated treatment efficacy for the last illness episode western treatment was generally perceived as more effective than traditional or faith healing. However, traditional or faith heal- 


\begin{tabular}{|l|l|l|}
\hline \multirow{2}{*}{ Question } & Traditional & Western \\
\cline { 2 - 3 } & $\mathrm{M}(\mathrm{SD})$ & $\mathrm{M}(\mathrm{SD})$ \\
\hline 1. How effective was the treatment? & $2.2(1.3)$ & $4.1(1.8)$ \\
2. Will remain feeling better? & $3.1(1.5)$ & $3.6(1.8)$ \\
3. Is this proof that the therapy works? & $3.4(1.5)$ & $3.9(1.7)$ \\
4. Worked because healer talked to him/her? & $2.7(1.6)$ & $1.8(1.3)$ \\
5. Worked because healer touched him/her? & $2.8(1.4)$ & $1.4(1.1)$ \\
6. The treatment itself worked? & $4.0(1.5)$ & $3.7(1.7)$ \\
7. Would have got better with time anyway? & $3.2(1.4)$ & $2.8(1.8)$ \\
\hline
\end{tabular}

$r=$ reverse scored

ing was considered almost as long lasting as western therapy. Major attributes for both traditional and western therapy was "the treatment itself worked". For traditional or faith healing "talking" and "touching" were considered more important for its treatment efficacy than for western therapy. It is also interesting to note that the majority rated "would have got better with time anyway" for both traditional $(M=3.2)$ and western $(M=2.8)$.

\section{Discussion}

The main aim of the study was to determine the public's perceived efficacy of traditional and western health care in the treatment of the last illness episode, of different degrees of severity. Most of the participants $(58 \%)$ had had some experience of one or more forms of traditional therapies, which concurs with other studies with African samples (Farrand 1984: 779f.). A large percentage (34\%) had consulted a traditional healer for the last illness episode. This confirms their important role in health care, even in this urban population.

Regarding the treatment of the last illness episode in this sample, the great majority of the participants, consulted western therapists. Western therapy was also clearly seen as more effective than traditional treatment. This was in agreement with previous work in this field (e.g. Peltzer 1981: 207, Vincent \& Furnham 1994:133).

Traditional as well as faith healing, on the other hand, was more often utilized in the treatment of traditional illnesses and to a lesser extent for some minor and chronic conditions, as found in some other studies (Furnham \& Bhagrath 1993: 240 , Peltzer 1987: 111). It is important to note that traditional and faith healing were considered as relatively long lasting and that 'talking' and 'touching' were seen as major attributes for treatment efficacy. These attributes of health care may indicate some weaknesses of western health care but also some strength of traditional healing. Important results from this study with reference to further work are that (1) people distinguish between different types of traditional and western therapies, (2) different therapies are perceived to be effective for different conditions: herbal treatment for minor conditions such as headache and body pains, divination for traditional illnesses, psychological therapy for more psychological conditions, faith healing for some chronic conditions such as cancer, medical therapy for e.g. major injury, eye problem, asthma, etc., and (3) a number of people are skeptical of the benefits of both traditional and western therapy saying that they "would have got better with time anyway" (cf. also Vincent and Furnham 1994: 133).

\section{Acknowledgement}

I am grateful for the funding of the research project by the University of the North. 
African National Congress 1994a. A national health plan for South Africa. Johannesburg: Author.

African National Congress 1994b. The Reconstruction and Development Programme. Johannesburg: Author.

Farrand, D. 1984. Is a combined western and traditional health service for black patients desirable? South African Medical Journal 66: 779-780.

Freeman, M. \& Motsei, M. 1992. Planning health care in South Africa: is there a role for traditional healers? Social Science \& Medicine 34: 1183-1090.

Furnham, A. \& Bhagrath, R. 1993. A comparison of health beliefs and behaviours of clients of orthodox and complementary medicine. British Journal of Clinical Psychology 32: 237-246

Hopa, M., Simbayi, L.C. \& Du Toit, C.D. 1998. Perceptions on integration of traditional and western healing in the new South Africa. South African Journal of Psychology 28: 8-14.

Louw, D.A. \& Pretorius, E. 1995. The traditional healer in a multicultural society: the South African experience. In: LL Adler \& BR Mukherji (eds.) Spirit versus scalpel: traditional healing and modern psychotherapy (pp. 41-58). London: Bergin \& Garrey.

Peltzer, K. 1981. Traditional medicine and its promotion in a Zimbabwean village. The Central African Journal of Medicine, 27: 205-208.

Peltzer, K. 1987. Traditional healing and mental health care in Malawi. Heidelberg: Asanger.

Peltzer, K. 1998a. A community survey of traditional healers in South Africa (Northern Province). South African Journal of Ethnology, 21: 191-197

Peltzer, K. 1998b. Traditional healing and primary health care in South Africa. In: CE Gottschalk-Batschkus \& C Raetsch (eds.) Ethnotherapies: therapeutic concepts in transcultural comparison (pp. 49-52). Berlin: WWB.

Peltzer, K. In print. Faith healing for mental and social disorders in the Northern Province (South Africa). Journal of Religion in Africa.

Pretorius, E. 1989. Skakeling tussen tradisionele en moderne geneeskunde in Afrika: die dekade sedert Alma Ata. Acta Academica 21: 101-129.

Swartz, L. 1986. Transcultural psychiatry in South Africa (Part 1). Transcultural Psychiatric Research Review 23: $273-303$.

Swartz, L. 1996. Culture and mental health in the rainbow nation: transcultural psychiatry in a changing South Africa. Transcultural Psychiatric Research Review 33: 229-136.

Vincent, C. \& Furnham, A. 1994. The perceived efficacy of complementary and orthodox medicine: preliminary findings and the development of a questionnaire. Complementary Therapies in Medicine 2: 128-134. 